\title{
Interactions between dietary oil treatments and genetic variants modulate fatty acid ethanolamides in plasma and body weight composition
}

\author{
Shuaihua $\mathrm{Pu}^{1,2}$, Peter Eck ${ }^{1}$, David J. A. Jenkins ${ }^{3,4}$, Philip W. Connelly ${ }^{5,6}$, Benoît Lamarche ${ }^{7}$, \\ Penny M. Kris-Etherton ${ }^{8}$, Sheila G. West ${ }^{8,9}$, Xiaoran Liu $^{8}$ and Peter J. H. Jones ${ }^{1,2 *}$ \\ ${ }^{1}$ Department of Human Nutritional Sciences, University of Manitoba, Winnipeg, MB R3T 2N2, Canada \\ ${ }^{2}$ Richardson Centre for Functional Foods and Nutraceuticals, University of Manitoba, Winnipeg, MB R3T 6C5, Canada \\ ${ }^{3}$ Department of Nutritional Sciences, Faculty of Medicine, University of Toronto, Toronto, ON M5S 3E2, Canada \\ ${ }^{4} \mathrm{Li}$ Ka Shing Knowledge Institute, St. Michael's Hospital, Toronto, ON M5B $1 \mathrm{~W} 8$, Canada \\ ${ }^{5}$ Keenan Research Centre for Biomedical Science, St. Michael's Hospital, Toronto, ON M5B 1W8, Canada \\ ${ }^{6}$ Departments of Medicine and Laboratory Medicine and Patbobiology, University of Toronto, Toronto, ON M5S 1A1, Canada \\ ${ }^{7}$ Institute of Nutrition and Functional Foods, Université Laval, Québec, QC G1V OA6, Canada \\ ${ }^{8}$ Department of Nutritional Sciences, Pennsylvania State University, University Park, PA 16802, USA \\ ${ }^{9}$ Department of Biobehavioral Health, Pennsylvania State University, University Park, PA 16802, USA
}

(Submitted 24 August 2015 - Final revision received 7 December 2015 - Accepted 10 December 2015 - First publisbed online 25 January 2016)

\section{Abstract}

Fatty acid ethanolamides (FAE), a group of lipid mediators derived from long-chain fatty acids (FA), mediate biological activities including activation of cannabinoid receptors, stimulation of fat oxidation and regulation of satiety. However, how circulating FAE levels are influenced by FA intake in humans remains unclear. The objective of the present study was to investigate the response of six major circulating FAE to various dietary oil treatments in a five-period, cross-over, randomised, double-blind, clinical study in volunteers with abdominal obesity. The treatment oils $(60 \mathrm{~g} / 12552 \mathrm{~kJ}$ per d $(60 \mathrm{~g} / 3000 \mathrm{kcal}$ per d) $)$ provided for $30 \mathrm{~d}$ were as follows: conventional canola oil, high oleic canola oil, high oleic canola oil enriched with DHA, flax/safflower oil blend and corn/safflower oil blend. Two SNP associated with FAE degradation and synthesis were studied. Post-treatment results showed overall that plasma FAE levels were modulated by dietary FA and were positively correlated with corresponding plasma FA levels; minor allele (A) carriers of SNP rs324420 in gene fatty acid amide hydrolase produced higher circulating oleoylethanolamide (OEA) $(P=0.0209)$ and docosahexaenoylethanolamide (DHEA) levels $(P=0 \cdot 0002)$. In addition, elevated plasma DHEA levels in response to DHA intake tended to be associated with lower plasma OEA levels and an increased gynoid fat mass. In summary, data suggest that the metabolic and physiological responses to dietary FA may be influenced via circulating FAE. Genetic analysis of rs324420 might help identify a sub-population that appears to benefit from increased consumption of DHA and oleic acid.

\section{Key words: Dietary oil treatments: Fatty acid ethanolamides: Intervention trial: Genetic variants: Body weight}

Fatty acid ethanolamides (FAE), also referred to as $\mathrm{N}$ acylethanolamines, are a group of endogenous ethanolamides of different fatty acids (FA) that were first identified in the late $1970 \mathrm{~s}^{(1)}$. $\mathrm{N}$-arachidonoylethanolamine (AEA, also called anandamide), the derivative of arachidonic acid (AA), is the first isolated and identified FAE compound ${ }^{(2)}$ and has been characterised in placenta and fetal membranes as well as human plasma, amniotic fluid ${ }^{(3)}$ and the nervous system ${ }^{(4)}$. AEA serves as an endogenous ligand of cannabinoid receptors $(\mathrm{CB})^{(5)}$. Oleoylethanolamide (OEA), a derivative of oleic acid (OA), is thought to regulate satiety and body weight $^{(())}$by activating PPAR- $\alpha$, which is responsible for energy expenditure and energy intake through lipolysis in adipocytes ${ }^{(7)}$. Consequently, administration of OEA may become part of the treatment of eating disorders and body weight maintenance ${ }^{(8)}$. Other FAE, including palmitoylethanolamide (PEA) and linoleoylethanolamide (LEA) over a range of concentrations are believed to have anti-inflammatory properties ${ }^{(9-11)}$. Little is known, however, about docosahexaenoylethanolamide (DHEA; derivative

Abbreviations: AA, arachidonic acid; AEA, $N$-arachidonoylethanolamine; ALA, $\alpha$-linolenic acid; ALEA, $\alpha$-linolenoylethanolamide; Canola, conventional canola oil; CanolaDHA, DHA-enriched canola oil; CanolaOleic, high oleic canola oil; CornSaff, a blend of corn oil and safflower oil; DHEA, docosahexaenoylethanolamide; FA, fatty acid; FAE, fatty acid ethanolamide; FAAH, fatty acid amide hydrolase; FlaxSaff, a blend of flax oil and safflower oil; LA, linoleic acid; LEA, linoleoylethanolamide; NAPE-PLD, $N$-acylphosphatidylethanolamine-hydrolysing phospholipase D; OA, oleic acid; OEA, oleoylethanolamide; PEA, palmitoylethanolamide.

* Corresponding author: P. J. Jones, fax +1 204474 7552, email Peter.Jones@umanitoba.ca 
of DHA) or $\alpha$-linolenoylethanolamide (ALEA; derivative of $\alpha$-linolenic acid (ALA)), although it was observed that a high dietary intake of DHA increased brain DHEA levels in piglets, suggesting a similar metabolism of AEA in the nervous system ${ }^{(12)}$. Recently, it has been reported that DHEA-dependent pathways may be associated with hippocampal neurodevelopment and synaptic activity ${ }^{(13)}$. However, the biological importance of $n$-3-derived FAE remains to be resolved.

The endogenous FAE are involved in numerous biological activities and primarily modulated by their biosynthesis and degradation ${ }^{(14)}$. Generally, the enzymes $\mathrm{N}$-acylphosphatidylethanolamine-hydrolysing phospholipase D (NAPE-PLD) and fatty acid amide hydrolase (FAAH) appear to be responsible for the synthesis and hydrolysis of FAE, respectively, in turn influencing circulating FAE levels in vivo ${ }^{(15,16)}$. With the growing knowledge of nutrigenomics, the common genetic variants of NAPE-PLD and FAAH genes may modulate circulating FAE levels in humans. Investigations of common SNP in humans have demonstrated that major allele carriers of SNP rs324420 of FAAH are more likely associated with overweight/obesity and metabolic disorders ${ }^{(17,18)}$, although the functional significance of these differences remain unclear over time ${ }^{(19,20)}$. It was proposed that this polymorphism might impact specific neural mechanisms through CB signalling which, in turn, increase the risk of reward deficiency syndrome that causes obesity ${ }^{(21)}$. Meanwhile, although little is known about NAPE-PLD, a study has shown that a common haplotype of the NAPE-PLD gene may be protective against obesity ${ }^{(22)}$. Most recently, Geurts et $a l^{(23)}$ proposed an important mechanism of adipose tissue NAPE-PLD on whole-body metabolism using NAPE-PLD knockout mice, indicating that NAPE-PLD may play an essential role in regulating energy homoeostasis and be responsive to cold-induced browning. In other words, alterations of the enzyme activity of NAPE-PLD may be beneficial as an anti-obesity treatment.

In addition, evidence has demonstrated that intake of highMUFA diets elicits weight loss and/or body fat mass reduction compared with consuming high-SFA diets in humans ${ }^{(24-27)}$. In addition, oral administration of OEA appeared to beneficially affect health, resulting in weight loss in humans ${ }^{(16,28)}$. Therefore, a knowledge gap exists concerning whether endogenous OEA, converted from dietary OA, plays a role on body fat distribution. In addition, it remains to be determined whether the consumption of FA other than oleic acid can contribute to changes in body fat composition via their corresponding FAE.

Therefore, the purpose of the present study was to answer the following questions; first, how major FAE shift in response to dietary oil treatments in humans; second, whether common genetic variants in FAAH and NAPE-PLD genes affect plasma FAE levels; third, whether possible diet-gene interactions exist in populations with risk of metabolic syndrome; and, finally, whether associations exist between changes in circulating FAE concentrations, especially OEA, and body fat composition after the dietary interventions. Therefore, we hypothesised that consumption of various dietary oil treatments with differing FA composition can lead to corresponding shifts in plasma FAE levels, and the diet-gene interaction may play an important role on the levels of FAE in response to dietary treatments, resulting in potential changes in body fat composition.

\section{Methods}

\section{Clinical design}

This study was conducted as part of the Canola Oil Multicenter Intervention Trial (COMIT) study, a dietary intervention in adults with abdominal obesity and at least one criterion for metabolic syndrome ${ }^{(29)}$. The COMIT study was conducted at the Richardson Centre for Functional Foods and Nutraceuticals (RCFFN) at the University of Manitoba, the Institute of Nutraceuticals and Functional Foods at the Laval University and the Department of Nutritional Sciences at the Pennsylvania State University between September 2010 and March 2012, as previously described $^{(30)}$. The study was registered at clinicaltrials. gov (NCT01351012). All the participants provided their written informed consent before the study started. The study was reviewed and approved by Institutional Review Boards or Committees at all participating sites.

The COMIT study investigated effects of a daily intake of $60 \mathrm{~g}$ of dietary oils low in SFA and high in MUFA or PUFA. A randomised cross-over study design was implemented, in which participants were randomly assigned to five novel vegetable oil treatments. Each treatment phase was $30 \mathrm{~d}$ in duration and was separated by a 4-week washout period. The diets were designed to maintain body weight, and energy needs for each participant were calculated using the Mifflin equation ${ }^{(31)}$. With the exception of the treatment oils, an identical 7-d rotating menu in the full-feeding diets was served across all treatments, with a fixed macronutrient composition (35\% fat, 50\% carbohydrate, $15 \%$ protein). The five oil treatments were as follows: canola oil (Canola; $60 \%$ OA, $20 \%$ linoleic acid (LA), 10\% ALA), high oleic acid canola oil (CanolaOleic; $72 \%$ OA, 15\% LA, 2\% ALA), DHA-enriched canola oil (CanolaDHA; 63\% OA, 13\% LA, $6 \%$ DHA (from an algal oil)), a blend of corn oil/safflower oil (CornSaff; 18\% OA, 69\% LA) and a blend of flax oil/safflower oil (FlaxSaff; 18\% OA, 38\% LA, 32\% ALA) (Table 1). Three canola-based diets were rich in MUFA, whereas the two safflower oil blends were high in $n$-3 PUFA or $n-6$ PUFA. Compliance with the feeding protocol was assessed by clinical coordinators who ensured that participants consumed at least one of their two treatment beverages under supervision daily, as well as evaluated the returned meal bags of non-consumed food provided for off-site consumption. Compliance was further confirmed by measuring post-treatment plasma FA profiles.

\section{Subjects}

A total of 170 volunteers were recruited using media advertisements. The inclusion criteria required an increased waist circumference ( $>94 \mathrm{~cm}$ for men and $>80 \mathrm{~cm}$ for women) or at least one of the following criteria: TAG $>1.7 \mathrm{mmol} / \mathrm{l}$; HDL-cholesterol $<1 \mathrm{mmol} / 1$ (men) or $<1.3 \mathrm{mmol} / 1$ (women); blood pressure $\geq 130 \mathrm{mmHg}$ (systolic) and/or $\geq 85 \mathrm{mmHg}$ (diastolic); and glucose $\geq 5.5 \mathrm{mmol} / 1$. Exclusion criteria included history of thyroid disease, diabetes mellitus, kidney disease and 
liver disease, current smokers, consuming more than two alcoholic drinks per week and taking lipid-lowering medications or supplements of any kind in the past 2 weeks.

\section{Blood sample collection}

Our intervention study was designed to compare end points after each experimental phase; therefore, the FA baseline composition profiles at each phase were not measured. Our aim was to systematically cross-compare responses across the treatment oil blends that were hypothesised to confer health benefits. After the 4-week washout to maintain their habitual diets and another 4-week treatment period, carry-over effects would be very limited, based on our previous clinical experience. Therefore, on days 29 and 30 of each phase, 12-h fasting blood samples were collected in EDTA-coated tubes and centrifuged at $3000 \mathrm{rpm}$ for $20 \mathrm{~min}$ at $4^{\circ} \mathrm{C}$. Separated plasma and leucocyte samples were transferred to labelled aliquot tubes and stored at $-80^{\circ} \mathrm{C}$ until further analysed. After the completion of the trial, samples for the present study were shipped to the RCFFN for analysis.

\section{Measurement of plasma fatty acid profiles}

Plasma FA concentrations were determined at the end of each experimental phase. Individual plasma FA classes were extracted from plasma-EDTA aliquots using the Folch method ${ }^{(32)}$, followed by methylation using methanolic hydrogen chloride as previously described $^{(30)}$. FA methyl esters were separated on a DB-225 column $(30 \mathrm{~m} \times 250 \mu \mathrm{m}$ with $0 \cdot 25-\mu \mathrm{m}$ film thickness; Agilent Technologies) using an Agilent $6890 \mathrm{~N}$ gas chromatograph equipped with a flame ionisation detector (GC-FID; Agilent Technologies). The oven was programmed from 70 to $240^{\circ} \mathrm{C}$ in five temperature steps $\left(70^{\circ} \mathrm{C}\right.$ for $1 \mathrm{~min}$, rise of $30^{\circ} \mathrm{C} / \mathrm{min}$ to $180^{\circ} \mathrm{C}$, rise of $10^{\circ} \mathrm{C} / \mathrm{min}$ to $200^{\circ} \mathrm{C}$, rise of $2^{\circ} \mathrm{C} / \mathrm{min}$ to $220^{\circ} \mathrm{C}$ and hold for $9.5 \mathrm{~min}$, rise of $40^{\circ} \mathrm{C} / \mathrm{min}$ to $240^{\circ} \mathrm{C}$ ). Samples were run with a $20: 1$ split ratio, and He was used as the carrier gas with a column flow rate of $1 \mathrm{ml} / \mathrm{min}$. Temperatures for the injector and detector

Table 1. Fatty acid composition of the five treatment oils $(60 \mathrm{~g}$ based on $12552 \mathrm{~kJ} \mathrm{diet} / \mathrm{d}(3000 \mathrm{kcal}$ diet/d))

\begin{tabular}{|c|c|c|c|c|c|}
\hline \multirow[b]{2}{*}{ Fat type (g) } & \multicolumn{5}{|c|}{ Treatments } \\
\hline & Canola & CanolaOleic & CanolaDHA & FlaxSaff & CornSaff \\
\hline SFA & 4.33 & 3.91 & $5 \cdot 19$ & 4.87 & 4.73 \\
\hline$c 16: 0$ & $2 \cdot 44$ & $2 \cdot 20$ & 3.15 & 2.94 & 3.52 \\
\hline$c 18: 0$ & $1 \cdot 10$ & $1 \cdot 10$ & 1.02 & 1.90 & 1.14 \\
\hline MUFA & 37.69 & $43 \cdot 19$ & 38.25 & $10 \cdot 72$ & $10 \cdot 60$ \\
\hline$c 18: 1 n-9$ & 35.17 & $42 \cdot 88$ & 37.95 & $10 \cdot 72$ & $10 \cdot 56$ \\
\hline PUFA & 17.58 & $10 \cdot 26$ & 13.97 & 41.67 & $41 \cdot 78$ \\
\hline$c 18: 2 n-6$ & 11.72 & 8.84 & 7.56 & $22 \cdot 48$ & 41.61 \\
\hline$c 18: 3 n-3$ & $5 \cdot 86$ & $1 \cdot 38$ & $1 \cdot 18$ & 19.19 & 0.17 \\
\hline$c 20: 4 n-6$ & 0 & 0 & 0.04 & 0 & 0 \\
\hline$c 20: 5 n-3$ & 0 & 0 & 0.09 & 0 & 0 \\
\hline$c 22: 5 n-3$ & 0 & 0 & 1.42 & 0 & 0 \\
\hline$c 22: 6 n-3$ & 0 & 0 & 3.48 & 0 & 0 \\
\hline
\end{tabular}

Canola, conventional canola oil; CanolaOleic, high oleic canola oil; CanolaDHA, DHA-enriched canola oil; FlaxSaff, a blend of flax oil and safflower oil; CornSaff, a blend of corn oil and safflower oil. were set at 280 and $300^{\circ} \mathrm{C}$, respectively. Individual FA were identified by comparison with GLC 461 standard (Nu-Chek Prep). The internal FA standard heptadecanoic acid (C17:0) (Sigma-Aldrich) was introduced into original plasma samples to quantify the amount of each FA in plasma. The proportion of substrate FA converted to FA products was calculated based on the peak area relative to the total area and expressed as the percentage of total FA.

\section{Total plasma fatty acid ethanolamide analysis}

Analysis of FAE was conducted using a previously described method $^{(33)}$. The six targeted FAE in plasma samples (PEA, OEA, LEA, AEA, ALEA and DHEA) were quantified according to the ratio of the known deuterated FAE in the internal standard mixture, with an expected relative standard deviation value of $<5 \%$. Standards including PEA, OEA, LEA, AEA, ALEA, DHEA, PEA-d4, OEA-d4, LEA-d4, AEA-d8 and DHEA-d4 were purchased from Cayman Chemicals Company. ALEA-d4 was synthesised in our laboratory using $\alpha$-linolenic acid chloride (Nu-Chek Prep) dissolved in methylene chloride with ethanolamide-d4 (Cambridge Isotope Laboratories) ${ }^{(2)}$. Relative ratios of FAE and their deuterated isotopes in the internal standard mixture were generated by serial dilution when the internal standard mixture was adjusted to the optimal concentration.

Aliquots of plasma samples at the end point of each phase were used for FAE extraction using a solid-phase extraction method $^{(34)}$ with some modifications. The stability of FAE in frozen plasma samples has been previously validated in our laboratory $^{(35,36)}$. In brief, plasma samples that were mixed with internal standards on ice were filtered under gentle vacuum at a low flow rate through an activated Oasis HLB cartridge (Waters Corporation). Extracts were then washed and eluted with acetonitrile, followed by analysis on an ultra-performance liquid chromatography (UPLC) tandem Quattro micro API mass spectrometer (Waters Corporation), as published by Lin et $a l^{(33)}$. A Kinetex XB-C18 column $(100 \times 2.1 \mathrm{~mm}, 1.7 \mu \mathrm{m}$; Phenomenex) was used for separation at a flow rate of $0 \cdot 2 \mathrm{ml} / \mathrm{min}$. Data were acquired and processed using MassLynx 4.1 (Waters Corporation).

\section{Measures of body fat mass}

Regional changes in body composition of study participants were assessed at both the initiation and termination of each dietary phase by dual-energy X-ray absorptiometry (DEXA) scanning according to the manufacturer's recommendations (GE Healthcare; QDR-4500W; Hologic Corp.). The equipment, room supplies and room set-up were checked on a regular basis. All maintenance work including calibration was performed according to the manufacturer's recommendations. Licensed DEXA technicians and coordinators performed all scans on each participant after each phase. Participants were required to lie down with the same posture each time at the centre of the measurement box from head to toe, face up and keep their arms at their sides during the scans. The regions of interest, including android fat mass, gynoid fat mass and the 
ratio of android:gynoid fat, were manually adjusted on the scan images. All data were then automatically calculated using the software Lunar Prodigy Advance enCORE and APEX System.

\section{SNP analysis}

To investigate the relationship between genetic variants of FAAH and NAPE-PLD genes and plasma FA and FAE levels, all the subjects were genotyped for two selected SNP (rs324420 for $F A A H$ and rs12540583 for NAPE-PLD). According to the GWAS and NCBI dbSNP database, the selected SNP both belong to missense mutations with a higher minor allele frequency that may lead to significant functional changes in the corresponding enzymes. Genomic DNA was extracted from leucocyte samples using a commercial Qiagen DNeasy Blood and Tissue Kit according to the manufacturer's instructions (Qiagen Sciences Inc.). The quality and quantity of individual genomic DNA were assessed by Thermo Scientific NanoDrop 2000 micro-volume spectrophotometer (Thermo Fisher Scientific). Amplification and detection of DNA were conducted with the 7500 Fast RealTime PCR System (Applied Biosystems, Life Technologies) using optical-grade 96-well plates. Each well contained $5 \mu \mathrm{l}$ of PCR reaction mixture, containing customised TaqMan SNP Genotyping Assays, TaqMan SNP genotyping Master Mix (Life Technologies Inc.) and pre-diluted DNA samples. Data were acquired by software StepOne 2.1 (Applied Biosystems, Life Technologies).

\section{Statistical analyses}

Only participants who completed all five dietary phases were included in the statistical analyses. A per-protocol approach was used to avoid the need for multiple imputations for missing data during the analyses. The power calculation was performed based on previous results on post-treatment plasma OEA levels in the clinical intervention by Jones et $a l^{(36)}$, and indicated a power of $100 \%(\alpha=0.05)$ to detect significant differences in OEA levels between three MUFA-rich diets and two PUFA-rich diets. Statistical analyses were performed using SAS 9.2 (SAS Inc.). Statistical significance was determined using the adjusted
Tukey test for multiple comparisons with $P<0 \cdot 05$. The results are expressed as least square means with their standard errors, unless noted elsewhere.

The effects of dietary treatment on plasma FA profiles, plasma FAE levels and body composition were analysed using a mixed model with repeated-measures analysis of time. Treatment, age and sex were considered as fixed factors. Sequence of treatments and centre were used as random effects in the model. Tukey-adjusted $P$ values were used to examine differences between-treatments. Pearson's correlation analyses were conducted to test for associations between plasma FA and FAE and between plasma FAE and fat mass change.

Effects of different genotypes on FAE levels were analysed using mixed models. Treatment, sex, age, genotype, genotype $\times$ sex interaction and treatment $\times$ genotype interaction were entered as fixed effects, sequence of treatments were random effects, and clinical sites were selected as random effects. Repeated measures by five dietary phases on subjects were used to investigate the effects of treatments, genotypes and their interactions. Two individual SNP were analysed separately.

\section{Results}

\section{Subject characteristics}

A total of 130 participants (sixty male; seventy female; $76.5 \%$ of the total randomised subjects) completed the five-phase intervention study. The mean ages of men and women were 46.5 (SD 14.2) and 47.6 (SD 14.5) years, with BMI of $29 \cdot 8$ (SD 4.4) and $29.0(\mathrm{sD} 4.2) \mathrm{kg} / \mathrm{m}^{2}$, respectively. Baseline characteristics of the study population have been previously published ${ }^{(30)}$ and are shown in Table 2 .

Plasma fatty acid profiles. The effects of the diet interventions on plasma FA profiles are summarised in Table 3. Overall, all changes in FA composition agreed with our expectation based on the FA profile of each intervention diet. The Canola and CanolaOleic diets produced the highest levels of total MUFA $(P<0.05)$, whereas CornSaff and FlaxSaff rich in $n-6$ and/or $n-3$ PUFA contents resulted in higher $(P<0.05)$ levels of PUFA compared with the other three diets. The CornSaff group

Table 2. Baseline characteristics of participants of the dietary intervention (Mean values with their standard errors; $n 130$ )

\begin{tabular}{|c|c|c|c|c|c|c|c|}
\hline \multirow[b]{2}{*}{ Characteristics } & \multicolumn{2}{|c|}{ Male $(n 60)$} & \multicolumn{2}{|c|}{ Female $(n 70)$} & \multicolumn{2}{|c|}{ Total $(n 130)$} & \multirow[b]{2}{*}{$P^{\star}$} \\
\hline & Mean & SEM & Mean & SEM & Mean & SEM & \\
\hline $\mathrm{BMI}\left(\mathrm{kg} / \mathrm{m}^{2}\right)$ & $30 \cdot 7$ & 0.6 & $29 \cdot 0$ & 0.5 & $29 \cdot 8$ & 4.3 & 0.0254 \\
\hline Age (years) & $45 \cdot 2$ & 1.8 & 47.6 & 1.7 & $46 \cdot 5$ & $14 \cdot 2$ & 0.3308 \\
\hline Total cholesterol $(\mathrm{mmol} / \mathrm{l})$ & $5 \cdot 2$ & 0.1 & 5.4 & 0.2 & $5 \cdot 3$ & $1 \cdot 1$ & 0.3560 \\
\hline HDL-cholesterol (mmol/l) & $1 \cdot 2$ & 0.0 & 1.4 & 0.0 & 1.3 & 0.3 & $<0.0001$ \\
\hline LDL-cholesterol (mmol/l) & $3 \cdot 1$ & 0.1 & 3.3 & 0.1 & 3.2 & 0.9 & 0.4276 \\
\hline TAG $(\mathrm{mmol} / \mathrm{l})$ & $2 \cdot 0$ & 0.2 & 1.6 & 0.1 & 1.8 & 1.0 & 0.0119 \\
\hline Glucose (mmol/l) & $5 \cdot 3$ & 0.1 & 5.4 & 0.2 & 5.4 & 1.1 & 0.8448 \\
\hline Body weight (kg) & $95 \cdot 1$ & 1.8 & 76.5 & 1.4 & $85 \cdot 1$ & $12 \cdot 8$ & $<0.0001$ \\
\hline Waist circumference $(\mathrm{cm})$ & $106 \cdot 6$ & 1.3 & 96.6 & 1.4 & $101 \cdot 2$ & $10 \cdot 9$ & $<0.0001$ \\
\hline Systolic BP $(\mathrm{mmHg})$ & 128.9 & $2 \cdot 2$ & 120.2 & $2 \cdot 0$ & $124 \cdot 3$ & $16 \cdot 7$ & 0.0041 \\
\hline Diastolic BP (mmHg) & 81.0 & 1.7 & $78 \cdot 6$ & $1 \cdot 3$ & $79 \cdot 7$ & 11.8 & 0.2659 \\
\hline
\end{tabular}

$\mathrm{BP}$, blood pressure

${ }^{*}$ ANOVA was used to analyse between-sex differences in continuous variables. Significant difference: $P<0.05$. 
Table 3. Selected plasma fatty acid profiles of participants at end points of each dietary phase $(\mathrm{g} / 100 \mathrm{~g})^{*}$ (Least square mean values with their standard errors; $n$ 130)

\begin{tabular}{|c|c|c|c|c|c|c|c|c|c|c|}
\hline \multirow[b]{3}{*}{ Fatty acids } & \multicolumn{10}{|c|}{ Treatments } \\
\hline & \multicolumn{2}{|c|}{ Canola } & \multicolumn{2}{|c|}{ CanolaOleic } & \multicolumn{2}{|c|}{ CanolaDHA } & \multicolumn{2}{|c|}{ FlaxSaff } & \multicolumn{2}{|c|}{ CornSaff } \\
\hline & Mean & SEM & Mean & SEM & Mean & SEM & Mean & SEM & Mean & SEM \\
\hline$c 16: 0$ & $27 \cdot 04^{\mathrm{b}}$ & 0.20 & $27 \cdot 40^{\mathrm{b}}$ & 0.20 & $28 \cdot 10^{\mathrm{a}}$ & 0.20 & $27 \cdot 41^{\mathrm{b}}$ & 0.20 & $27 \cdot 35^{\mathrm{b}}$ & 0.20 \\
\hline$c 18: 0$ & $11.83^{c}$ & $0 \cdot 10$ & $11.79^{c}$ & $0 \cdot 10$ & $12 \cdot 28^{b}$ & $0 \cdot 10$ & $12 \cdot 51^{\mathrm{a}}$ & 0.10 & $12 \cdot 34^{\mathrm{a}, \mathrm{b}}$ & $0 \cdot 10$ \\
\hline$c 18: 1 n-9$ & $14.90^{\mathrm{b}}$ & 0.19 & $15 \cdot 52^{\mathrm{a}}$ & 0.19 & $13 \cdot 36^{\mathrm{c}}$ & 0.19 & $12 \cdot 10^{d}$ & 0.18 & $11 \cdot 62^{d}$ & 0.18 \\
\hline$c 18: 2 n-6$ & $22.00^{\mathrm{C}}$ & 0.23 & $21.52^{\mathrm{c}}$ & 0.23 & $18.68^{\mathrm{d}}$ & 0.23 & $25 \cdot 13^{\mathrm{b}}$ & 0.23 & $25 \cdot 93^{\mathrm{a}}$ & 0.23 \\
\hline$c 18: 3 n-3$ & $0.79^{b}$ & 0.03 & $0.63^{c}$ & 0.03 & $0.57^{\mathrm{c}, \mathrm{d}}$ & 0.03 & $1.61^{\mathrm{a}}$ & 0.03 & $0.49^{d}$ & 0.03 \\
\hline$c 20: 4 n-6$ & $9 \cdot 28^{b}$ & 0.15 & $9 \cdot 67^{a}$ & 0.15 & $9 \cdot 70^{\mathrm{a}}$ & 0.15 & $8 \cdot 27^{c}$ & 0.15 & $9.59^{a, b}$ & 0.15 \\
\hline$c 20: 5 n-3$ & $1.09^{b}$ & 0.04 & $0.86^{c}$ & 0.04 & $1.53^{\mathrm{a}}$ & 0.04 & $1.45^{\mathrm{a}}$ & 0.04 & $0.49^{d}$ & 0.04 \\
\hline$c 22: 5 n-3$ & $0.81^{b}$ & 0.03 & $0.72^{\mathrm{C}}$ & 0.03 & $0.34^{\mathrm{e}}$ & 0.03 & $0.97^{\mathrm{a}}$ & 0.03 & $0.62^{d}$ & 0.03 \\
\hline$c 22: 6 n-3$ & $2 \cdot 84^{\mathrm{b}}$ & 0.09 & $2 \cdot 79^{\mathrm{b}}$ & 0.09 & $7 \cdot 21^{\mathrm{a}}$ & 0.10 & $2.59^{\mathrm{b}}$ & 0.09 & $2 \cdot 66^{\mathrm{b}}$ & 0.09 \\
\hline Total SFA & $41.74^{\mathrm{c}}$ & 0.19 & $41.92^{\mathrm{C}}$ & 0.19 & $43 \cdot 28^{a}$ & 0.19 & $42 \cdot 71^{\mathrm{a}, \mathrm{b}}$ & 0.19 & $42 \cdot 51^{\mathrm{b}}$ & 0.19 \\
\hline Total MUFA & $18 \cdot 05^{a}$ & 0.22 & $18 \cdot 50^{\mathrm{a}}$ & 0.22 & $16 \cdot 20^{\mathrm{b}}$ & 0.22 & $14 \cdot 78^{\mathrm{C}}$ & 0.22 & $14.49^{\mathrm{C}}$ & 0.22 \\
\hline Total PUFA & $40 \cdot 21^{b}$ & 0.22 & $39.54^{\mathrm{c}}$ & 0.22 & $40 \cdot 47^{b}$ & 0.22 & $42 \cdot 57^{\mathrm{a}}$ & 0.22 & $43.04^{\mathrm{a}}$ & 0.22 \\
\hline Total $n-6$ PUFA & $34.67^{c}$ & 0.23 & $34.46^{\mathrm{c}}$ & 0.23 & $30.92^{d}$ & 0.23 & $35.92^{\mathrm{b}}$ & 0.23 & $38.79^{a}$ & 0.23 \\
\hline Total $n$-3 PUFA & $5.53^{\mathrm{c}}$ & $0 \cdot 10$ & $5.03^{d}$ & 0.10 & $9 \cdot 62^{\mathrm{a}}$ & 0.11 & $6 \cdot 64^{\mathrm{b}}$ & $0 \cdot 10$ & $4.24^{\mathrm{e}}$ & $0 \cdot 10$ \\
\hline
\end{tabular}

showed the lowest $(P<0.05)$ ALA, EPA and DPA levels compared with the other four treatments. Post-treatment DHA concentrations were higher $(P<0.0001)$ after the CanolaDHA treatment than after the other four treatments, whereas no differences were observed across the other four treatments, indicating good dietary compliance across all centres.

Total plasma fatty acid ethanolamides. The end point concentrations of OEA, AEA, PEA, LEA, ALEA and DHEA ( $n$ 121) were successfully measured and are shown in Fig. 1. Samples from nine participants were excluded from this analysis because of failures in the extraction of samples being run through the cartridges or in the FAE measurement using UPLC. Overall, plasma PEA and OEA were the two major FAE. No differences were observed in plasma PEA levels across the five dietary treatments. Three MUFA-rich treatments - Canola, CanolaDHA and CanolaOleic - resulted in higher $(P<0.05)$ OEA levels compared with the two PUFA-rich diets - CornSaff and FlaxSaff. However, despite the similar OA content of the three canola-based diets, the CanolaDHA treatment appeared to lower the OEA levels compared with the CanolaOleic diet $(P<0 \cdot 05)$. The plasma AEA level after the CanolaOleic diet was higher $(P<0.05)$ compared with the CanolaDHA, CornSaff and FlaxSaff diets, but no difference was observed between the CanolaOleic and Canola treatments. The two PUFA-rich diets had higher $(P<0.05)$ LEA levels compared with the CanolaDHA and CanolaOleic treatments. The FlaxSaff diet had the highest $(P<0.0001)$ ALEA levels and the DHEA level was substantially higher $(P<0.0001)$ in response to the CanolaDHA diet compared with the other four diets.

\section{Correlation between plasma fatty acid ethanolamides and plasma fatty acids}

Pearson's correlation coefficients between plasma FAE and their corresponding FA are presented in Table 4. Overall, positive correlations existed between the five major FAE and their individual precursor FA $(P<0.0001)$, whereas DHEA-DHA $(r \quad 0.52 ; \quad P<0.0001)$ and ALEA-ALA $(r \quad 0.51 ; \quad P<0.0001)$ showed the strongest correlations. Only AEA failed to produce a significant association with AA $(r 0.04 ; P=0.3547)$.

\section{Body composition and correlation with plasma fatty acid ethanolamides}

A subgroup of twenty-seven (male $n 7$; female $n$ 20) volunteers at RCFFN completed all the baseline and end point DEXA measures at each phase, and therefore their data were used for the exploratory analysis on the correlations between body composition and plasma FAE levels. The baseline characteristics of the subjects are presented in online supplementary Table S1. Total fat mass, android fat mass, gynoid fat mass and the ratio of android:gynoid fat mass at baseline, end point and changes in each dietary phase are reported in Table 5. No significant differences were observed except for the end point gynoid fat mass $(P=0.0503)$. It was mostly attributed to higher $(P<0.05)$ gynoid fat mass for CanolaDHA compared with CanolaOleic. Changes of gynoid fat mass from baseline tended to differ $(P=0 \cdot 0803)$, due to an increase after CanolaDHA and a decrease after CanolaOleic.

Correlations between end point FAE levels and android fat mass change (end point $v$. baseline) were tested in the subset ( $n$ 27) using Pearson's correlations (Table 6). Although we noticed that this subgroup had an unbalanced sex ratio, no differences on android fat mass change were observed. Overall, negative correlations between plasma OEA $(r-0 \cdot 24 ; P=0.0049)$ (Fig. 2), AEA $(r-0.24 ; P=0.0059)$ and LEA $(r-0.20 ; P=0.0225)$ levels and android fat mass changes across five diets were observed. Moreover, similar negative correlations were observed between the OEA:DHEA ratio and android fat mass change $(r-0.20 ; P=0.0187)$. No correlations were observed between ALEA, DHEA or PEA and android fat mass change. 
(A)

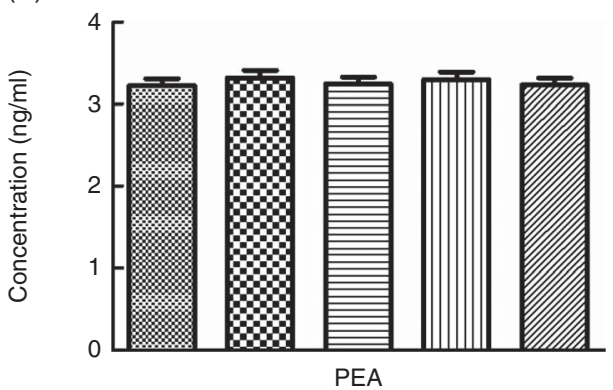

(C)

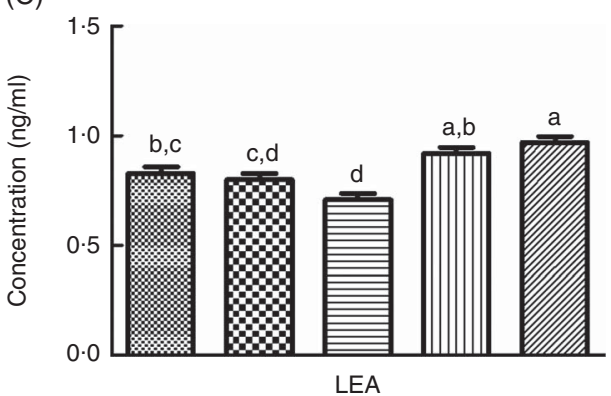

(E)

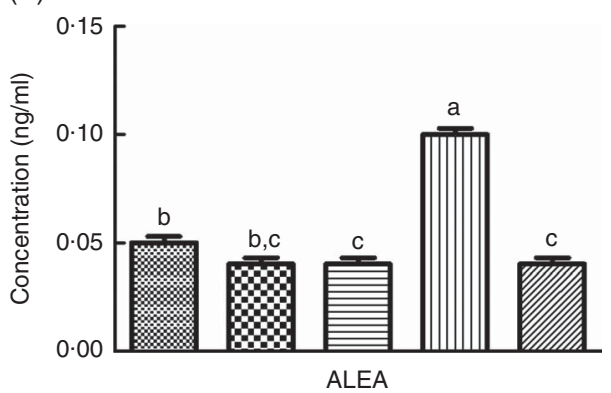

(B)

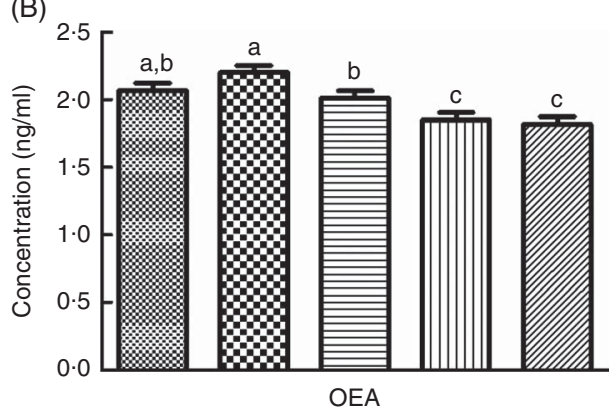

(D)

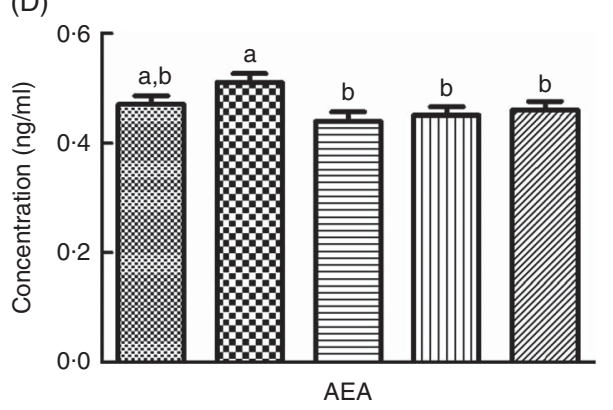

(F)

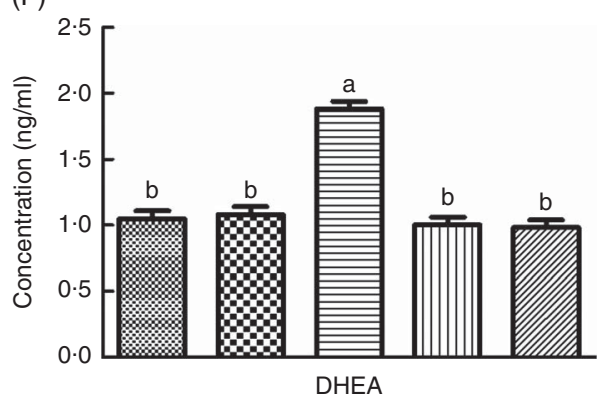

Fig. 1. Total plasma fatty acid ethanolamide levels at the end point of five dietary treatments. Values are presented in ng/ml as least squares means ( $n 121)$, with their standard errors. ${ }^{a, b, c}$ Mean values within each graph indicate significant differences between-treatments $(P<0.05)$. (A) Palmitoylethanolamide (PEA); (B) oleoylethanolamide $(\mathrm{OEA})$; (C) linoleoylethanolamide (LEA); (D) arachidonoylethanolamide (AEA); (E) a-linolenoylethanolamide (ALEA); (F) docosahexaenoylethanolamide (DHEA). 1 , Conventional canola oil; $\mathbf{Q}$, high oleic canola oil;

Table 4. Pearson's correlation between fatty acid ethanolamides (FAE) and their corresponding precursor fatty acids in plasma $(n 121)$

\begin{tabular}{llcr}
\hline Fatty acids & FAE & Correlation coefficient & \multicolumn{1}{c}{$P$} \\
\hline PA & PEA & 0.34504 & $<0.0001$ \\
OA & OEA & 0.36434 & $<0.0001$ \\
LA & LEA & 0.18462 & $<0.0001$ \\
AA & AEA & 0.03785 & 0.3547 \\
ALA & ALEA & 0.51068 & $<0.0001$ \\
DHA & DHEA & 0.51879 & $<0.0001$ \\
\hline
\end{tabular}

PA, palmitic acid; PEA, palmitoylethanolamide; OA, oleic acid; OEA, oleoylethanolamide; LA, linoleic acid; LEA, linoleoylethanolamide; AA, arachidonic acid; AEA, arachidonoylethanolamine; ALA, $a$-linolenic acid; ALEA, $a$-linolenoylethanolamide; DHEA, docosahexaenoylethanolamide.

\section{SNP characteristics and association with fatty acid ethanolamides}

Genomic DNA was genotyped for 129 of 130 subjects (one blood sample failed to provide enough DNA yield).
Analyses of the two SNP rs12540583 in NAPE-PLD and rs324420 in FAAH are summarised in Table 7.

As numbers of rare homozygotes in both polymorphisms were relatively low, the genotypes were grouped into two categories common homozygous and minor allele carriers. Results of the NAPE-PLD polymorphism rs12540583 (Table 8(a)) showed no differences in FAE levels across all treatments, except that the C-allele carriers had a higher $(P=0 \cdot 0010)$ DHEA levels after the CanolaDHEA diet, whereas a strong treatment $\times$ genotype interaction for DHEA was also observed $(P=0 \cdot 0004)$. In general, analyses of polymorphism rs324420 in FAAH (Table 8(b)) indicated that the A-allele carriers had higher $(P=0.0002)$ DHEA levels than the CC genotype carrier. In particular, after the Canola and CanolaDHA treatments, DHEA levels of A-allele carriers were higher $(P<0.05)$ than the homozygous $\mathrm{C}$ genotype group. In addition, a strong treatment $\times$ genotype interaction for DHEA was also observed $(P<0 \cdot 0001)$. DHEA levels after the CanolaDHA diet were observed to be higher in both genotypes compared with other treatments, and were significantly different between two 
Table 5. Body fat mass composition at the beginning and the end and changes of each dietary phase* (Least square mean values with their standard errors; $n 27$ (7, males; 20, females) from Richardson Centre for Functional Foods and Nutraceuticals)

\begin{tabular}{|c|c|c|c|c|c|c|c|c|c|c|c|}
\hline \multirow[b]{2}{*}{ Body fat profile } & \multicolumn{2}{|c|}{ Canola } & \multicolumn{2}{|c|}{ CanolaOleic } & \multicolumn{2}{|c|}{ CanolaDHA } & \multicolumn{2}{|c|}{ FlaxSaff } & \multicolumn{2}{|c|}{ CornSaff } & \multirow[b]{2}{*}{$P$} \\
\hline & Mean & SEM & Mean & SEM & Mean & SEM & Mean & SEM & Mean & SEM & \\
\hline \multicolumn{12}{|l|}{ Baseline } \\
\hline Total fat mass $(\mathrm{kg})$ & 33.72 & $2 \cdot 10$ & 33.35 & $2 \cdot 10$ & 33.40 & $2 \cdot 10$ & 33.60 & $2 \cdot 10$ & 33.49 & $2 \cdot 10$ & 0.6231 \\
\hline Android fat mass $(\mathrm{kg})$ & 3.42 & 0.22 & 3.37 & 0.22 & 3.40 & 0.22 & 3.41 & 0.22 & 3.39 & 0.22 & 0.8205 \\
\hline Gynoid fat mass (kg) & 5.61 & 0.43 & 5.58 & 0.43 & 5.56 & 0.43 & 5.57 & 0.43 & 5.57 & 0.43 & 0.9653 \\
\hline Android:gynoid ratio & 0.63 & 0.03 & 0.62 & 0.03 & 0.63 & 0.03 & 0.63 & 0.03 & 0.63 & 0.03 & 0.7659 \\
\hline \multicolumn{12}{|l|}{ End point } \\
\hline Total fat mass $(\mathrm{kg})$ & $33 \cdot 11$ & 2.08 & $32 \cdot 81$ & 2.08 & $33 \cdot 13$ & 2.08 & 32.97 & 2.08 & $33 \cdot 28$ & 2.08 & 0.2496 \\
\hline Android fat mass $(\mathrm{kg})$ & 3.39 & 0.22 & 3.33 & 0.22 & 3.39 & 0.22 & 3.36 & 0.22 & 3.40 & 0.22 & 0.2285 \\
\hline Gynoid fat mass (kg) & 5.47 & 0.41 & 5.43 & 0.41 & 5.63 & 0.41 & 5.47 & 0.41 & 5.55 & 0.41 & 0.0503 \\
\hline Android:gynoid ratio & 0.63 & 0.03 & 0.63 & 0.03 & 0.62 & 0.03 & 0.63 & 0.03 & 0.63 & 0.03 & 0.3273 \\
\hline \multicolumn{12}{|l|}{ Changes } \\
\hline Total fat mass $(\mathrm{kg})$ & -0.42 & 0.23 & -0.15 & 0.23 & -0.05 & 0.23 & -0.49 & 0.23 & -0.23 & 0.23 & 0.4703 \\
\hline Android fat mass $(\mathrm{kg})$ & -0.02 & 0.03 & -0.04 & 0.03 & 0.01 & 0.03 & -0.07 & 0.03 & -0.01 & 0.03 & 0.3848 \\
\hline Gynoid fat mass $(\mathrm{kg})$ & $-0 \cdot 11$ & 0.06 & $-0 \cdot 11$ & 0.06 & 0.06 & 0.06 & 0.01 & 0.06 & -0.06 & 0.06 & 0.0803 \\
\hline Android:gynoid ratio & 0.00 & 0.01 & 0.01 & 0.01 & -0.01 & 0.01 & -0.01 & 0.01 & 0.00 & 0.01 & 0.1464 \\
\hline
\end{tabular}

Canola, conventional canola oil; CanolaOleic, high oleic canola oil; CanolaDHA, DHA-enriched canola oil; FlaxSaff, a blend of flax oil and safflower oil; CornSaff, a blend of corn oil and safflower oil.

* Mixed-model ANOVA corrected by sex and post hoc Tucky's test were used to analyse treatment effects on different fat mass profiles. Significant difference: $P<0.05$.

Table 6. Pearson's correlation between end point fatty acid ethanolamide (FAE) levels and android fat mass changes of each dietary treatment ( $n$ 27).

\begin{tabular}{lcc}
\hline FAE & Correlation coefficients & $P^{*}$ \\
\hline PEA & -0.14 & 0.1037 \\
OEA & -0.24 & 0.0049 \\
LEA & -0.20 & 0.0225 \\
AEA & -0.24 & 0.0059 \\
ALEA & -0.08 & 0.3564 \\
DHEA & 0.08 & 0.3564 \\
OEA:DHEA ratio & -0.20 & 0.0187 \\
\hline PEA, palmitoylethanolamide; OEA, oleoylethanolamide; LEA, linoleoylethanolamide; AEA, \\
arachidonoylethanolamine; ALEA, a-linolenoylethanolamide; DHEA, docosahexaenoy- \\
lethanolamide.
\end{tabular}

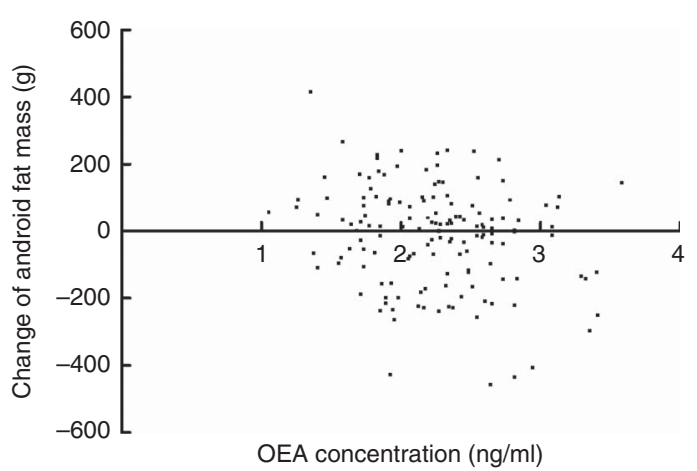

Fig. 2. The correlation between oleoylethanolamide (OEA) and change of android fat mass (end point $v$. baseline) across all five diets ( $n$ 27). A weak negative but significant correlation was observed $(r-0.24 ; P=0.0049)$.

genotypes. No differences were observed in the corresponding plasma DHA levels between the two genotypes.

\section{Discussion}

The present study demonstrated that circulating FAE levels reflected their precursor FA from the dietary intake, under controlled feeding conditions. Polymorphisms of rs324420 $(F A A H)$ may play a role in modulating the circulating levels of OEA and DHEA, which might further influence the body fat mass distribution. Given the growing evidence for the importance of FAE in governing energy metabolism, the implications of these trial results could be substantial.

Accumulating evidence suggests that circulating FAE levels are influenced by genetic variants, which account for the FAE synthesis and degradation in vivo ${ }^{(37)}$. In this study, we have extended the genetic investigation by examining the differences among end point plasma FAE in response to various dietary FA. Our results for the polymorphism rs12540583 with a missense mutation in humans also indicated a very limited effect of NAPE-PLD mutations on circulating FAE levels, except for the CanolaDHA treatment. To the best of our knowledge, this is the first study that has reported an analysis of the association between this polymorphism and circulating FAE levels. One clinical study reported that a common haplotype in NAPE-PLD was protective against obesity ${ }^{(22)}$, but no other human studies have assessed whether NAPE-PLD deficiency influences circulating FAE. In animal models, it has been reported that FAE levels were unaltered in NAPE-PLD-deficient mice ${ }^{(38)}$, suggesting that FAE might be synthesised by both NAPEPLD-dependent and NAPE-PLD-independent pathways based on the NAPE. Recently, Geurts et al. ${ }^{(23)}$ reported approximately $60 \%$ reduction of PEA, OEA and stearoylethanolamine levels in the adipose tissues, but not in the brain, of NAPE-PLD-deleted mice compared with wild-type mice when AEA remained unchanged in both genotypes. The important evidence on research of the NAPE-PLD gene suggests the existence of an alternative pathway of FAE synthesis ${ }^{(39)}$. Interestingly, the lack of positive correlation between circulating AA and AEA in the present study suggests that the similar alternative synthesis pathway of AEA may exist in humans. These data also suggest that plasma AA may not directly reflect the amount in the AEA precursor pool, because AA levels can also be affected by the 
Table 7. Characteristics of the selected SNP associated with metabolism of fatty acid ethanolamides ( $n$ 129).

\begin{tabular}{lllcrcc}
\hline & & & & \multicolumn{3}{c}{ Genotype } \\
\cline { 3 - 7 } SNP & Gene & Annotation & Alleles (major/minor) & MM & Mm & mm \\
\hline rs12540583 & NAPE-PLD & Missense & A/C & 103 & 25 & 1 \\
rs324420 & FAAH & Missense & C/A & 85 & 39 & 5 \\
\hline
\end{tabular}

MM, homozygous for the major allele; Mm, heterozygous; mm, homozygous for the minor allele; MAF, minor allele frequency; $N A P E-P L D, N$-acyl phosphatidylethanolamine phospholipase D; $F A A H$, fatty acid amide hydrolase.

level of its precursor, LA, and the levels of its derivatives, eicosanoids.

Apart from synthesis of FAE, the regulation of FAE levels also involves degradation. Enzymatic hydrolysis can degrade FAE to FA and ethanolamine by FAAH in virtually all mammalian tissues $^{(40)}$. FAAH is an integral membrane enzyme that can catalyse the hydrolysis of all FAE. As such, genetic variants of the $F A A H$ gene could alter the functionality of enzyme activity. The genetic missense polymorphism rs324420 in the FAAH gene has been extensively studied as an endocannabinoid risk factor in overweight/obesity and for potential cannabinoid antagonist treatment in obese populations ${ }^{(17)}$. However, the direct association between this SNP and obesity has not been evaluated. One study in overweight/obese subjects with binge eating disorder (BED) or non-BED demonstrated a positive correlation between A-allele carriers of rs324420 with overweight/obesity people, but no differences between BED and non-BED subjects ${ }^{(18)}$. In addition, it was reported that the polymorphism rs324420 variants may be associated with earlyonset but not adult obesity ${ }^{(41)}$. Our study did not show any direct association between rs324420 and BMI types (data not shown). In the present study, the A-allele carriers of rs324420 in $F A A H$ had a higher circulating DHEA level. It suggests that the A-allele carriers may have a lower activity rate for hydrolysing DHEA, resulting in a relatively higher level of DHEA, especially in response to consumption of DHA-enriched diets. Interestingly, such diet-gene interactions were observed only for DHEA and ALEA, indicating this SNP rs324420 may only affect DHEA and ALEA levels. Although little is known about the potential bioactive compounds DHEA and ALEA, studies have reported that the beneficial functions of DHA or DHEA could be attributed to major DHEA derivatives, which both have potent anti-inflammatory and organ-protective properties ${ }^{(42,43)}$. As such, these findings concerning the SNP rs324420 may be useful for further research designed to examine the beneficial effects of DHA consumption.

On the basis of animal studies, OEA is of pharmaceutical interest because of the effects it has on the endocannabinoid system, which could be a therapy for treating severe obesity by inhibiting appetite. Recently, a study compared the efficacy for anti-obesity treatment using orally administered OEA and rimonabant, an anorectic anti-obesity drug approved for use in Europe $^{(6)}$. Results indicated that OEA might represent a novel alternative to cannabinoid antagonists for the control of appetite, and rimonabant might also affect food intake and share a similar mechanism. Our results demonstrate a negative correlation between circulating OEA levels and the change in android fat mass from baseline across all five diets. This finding indicates that endogenous OEA from dietary OA might play a role in reducing fat mass. It is possible that the reduction of body weight, especially gynoid fat mass, by consuming diets high in OA is due to OEA: (a) suppression of appetite or (b) increased fat oxidation, resulting in decreased food intake. However, in our study, food intake was controlled and subjects on all diets consumed the same number of energy content across phases ${ }^{(30)}$. Thus, in the present study, our observations may be due to a different mechanism(s). Nevertheless, a different study conducted by our group showed a trend for increasing food intake and body weight in response to consumption of a DHA-enriched high oleic canola oil compared with corn oil or high oleic canola oil in golden hamsters ${ }^{(35)}$. In the present study, the CanolaDHA diet did not reduce android fat mass, but increased gynoid fat mass compared with the other two oleic acid-rich diets, resulting in the lowest total fat mass change. Consistent findings about the OEA:DHEA ratio change have been reported in $\mathrm{CD} 1$ mice $^{(44)}$, whereas our results reported lower plasma OEA levels in response to the DHA-rich diet. Recent evidence showed that DHEA might be a potent mediator for neurogenic differentiation ${ }^{(45)}$, and plays a role in reduction of headache and psychological distress in humans ${ }^{(46)}$, although we are not aware how such bioactivity of DHEA on neurological system could influence the OEA metabolism. Therefore, our observation provides indirect evidence to support the discovery of the effects of FAE on appetite and satiety that has been reported by Piomelli et $a l .{ }^{(47)}$. Moreover, we speculate that the elevation of DHEA in response to dietary DHA interferes with the synthesis and function of OEA to potentially suppress appetite and food intake, which would prevent weight loss.

In the present study, the plasma FA profile reflected the dietary FA in the treatment oils. The good compliance of participants across the three clinical sites has been previously reported ${ }^{(30)}$. As expected, corresponding elevations in end point FAE levels were also observed after the dietary interventions. Our results indicate that individuals with elevated plasma FA levels tend to have higher corresponding FAE levels. Results of a clinical study by Joosten et al. ${ }^{(48)}$ agreed with our findings and further suggest that the NEFA level may be positively correlated with both circulating fasting or non-fasting FAE. Thus, these NEFA molecules from increased membrane phospholipid cleavage become precursors of endogenous FAE as well. It is possible that the influences of NEFA on circulating FAE are based on their mobilisation from adipose tissue and clearance in the fasted state. Nevertheless, no further evidence would support the physiological stimuli involved in the changes of NEFA levels after meal consumption. In addition, as FAE are mostly converted in the tissues and then released into the 
Table 8. Selected plasma fatty acid ethanolamides (FAE) at the end point of five dietary treatments, by rs12540583 in $N$-acyl phosphatidylethanolamine phospholipase $\mathrm{D}(N A P E-P L D)$ and rs 324420 in fatty acid amide hydrolase $(F A A H)^{*}$

(Least square mean values; $n$ 120)

\begin{tabular}{|c|c|c|c|c|c|c|c|}
\hline \multirow[b]{2}{*}{ FAE } & \multicolumn{7}{|c|}{ Treatments } \\
\hline & Genotype & Canola & CanolaOleic & CanolaDHA & FlaxSaff & CornSaff & Overall \\
\hline \multicolumn{8}{|c|}{ (a) rs12540583 in NAPE-PLD } \\
\hline \multirow[t]{4}{*}{ PEA (ng/ml) } & AA & 3.2538 & 3.3977 & $3 \cdot 2641$ & 3.3363 & 3.2801 & 3.3064 \\
\hline & $\mathrm{AC} / \mathrm{CC}$ & 3.0356 & 2.9576 & $3 \cdot 1141$ & $3 \cdot 1249$ & 3.0344 & 3.0533 \\
\hline & $P$ & 0.9867 & 0.4697 & 0.9992 & 0.9892 & 0.9686 & 0.1111 \\
\hline & $P_{\text {interaction }}$ & & & & & & 0.2031 \\
\hline \multirow[t]{4}{*}{$\mathrm{OEA}(\mathrm{ng} / \mathrm{ml})$} & AA & $2 \cdot 0240$ & $2 \cdot 2076$ & $2 \cdot 0125$ & $1 \cdot 8198$ & 1.8262 & 1.9780 \\
\hline & $\mathrm{AC} / \mathrm{CC}$ & $2 \cdot 1030$ & 2.0432 & 1.8884 & 1.8099 & 1.6429 & 1.8975 \\
\hline & $P$ & 0.9999 & 0.9764 & 0.9968 & 1.0000 & 0.9507 & 0.4809 \\
\hline & $P_{\text {interaction }}$ & & & & & & 0.2144 \\
\hline \multirow[t]{4}{*}{ LEA (ng/ml) } & AA & 0.7924 & 0.8027 & $0 \cdot 7166$ & 0.8927 & 0.9557 & 0.8320 \\
\hline & $\mathrm{AC} / \mathrm{CC}$ & 0.9368 & 0.7444 & 0.6671 & 0.9842 & 0.9873 & 0.8640 \\
\hline & $P$ & 0.6667 & 0.9990 & 0.9997 & 0.9712 & 1.0000 & 0.5756 \\
\hline & $P_{\text {interaction }}$ & & & & & & 0.0411 \\
\hline \multirow[t]{4}{*}{ AEA (ng/ml) } & AA & 0.4790 & 0.5288 & 0.4522 & 0.4610 & 0.4713 & 0.4785 \\
\hline & $\mathrm{AC} / \mathrm{CC}$ & 0.4450 & 0.4379 & 0.4024 & 0.4189 & 0.4134 & 0.4235 \\
\hline & $P$ & 0.9960 & 0.2991 & 0.9403 & 0.9808 & 0.8616 & 0.0566 \\
\hline & $P_{\text {interaction }}$ & & & & & & 0.6246 \\
\hline \multirow[t]{4}{*}{ ALEA (ng/ml) } & AA & 0.0524 & 0.0442 & 0.0423 & 0.0997 & 0.0408 & 0.0559 \\
\hline & $\mathrm{AC} / \mathrm{CC}$ & 0.0519 & 0.0395 & 0.0363 & 0.1103 & 0.0401 & 0.0556 \\
\hline & $P$ & 1.0000 & 0.9998 & 0.9978 & 0.9036 & 1.0000 & 0.9543 \\
\hline & $P_{\text {interaction }}$ & & & & & & 0.3218 \\
\hline \multirow[t]{4}{*}{ DHEA (ng/ml) } & AA & 1.0434 & 1.0953 & 1.7738 & 1.0146 & 0.9678 & $1 \cdot 1790$ \\
\hline & $\mathrm{AC} / \mathrm{CC}$ & $1 \cdot 1485$ & 1.0234 & $2 \cdot 2034$ & 1.0211 & 1.0457 & 1.2884 \\
\hline & $P$ & 0.9903 & 0.9995 & 0.0010 & 1.0000 & 0.9989 & 0.1141 \\
\hline & $P_{\text {interaction }}$ & & & & & & 0.0004 \\
\hline \multicolumn{8}{|c|}{ (b) rs324420 in FAAH } \\
\hline \multirow{4}{*}{ PEA (ng/ml) } & CC & 3.0678 & $3 \cdot 1518$ & $3 \cdot 1416$ & $3 \cdot 1820$ & 3.1172 & $3 \cdot 1321$ \\
\hline & CA/AA & 3.5722 & 3.6652 & 3.4963 & 3.5806 & 3.5045 & 3.5638 \\
\hline & $P$ & 0.2108 & 0.1865 & 0.7054 & 0.5551 & 0.5930 & 0.0067 \\
\hline & $P_{\text {interaction }}$ & & & & & & 0.8483 \\
\hline \multirow[t]{4}{*}{ OEA (ng/ml) } & $\mathrm{CC}$ & 1.9085 & 2.0534 & 1.9521 & 1.7424 & 1.7156 & 1.8744 \\
\hline & CA/AA & $2 \cdot 2849$ & $2 \cdot 3984$ & $2 \cdot 0372$ & 1.9480 & 1.9020 & $2 \cdot 1141$ \\
\hline & $P$ & 0.0826 & $0 \cdot 1527$ & 0.9996 & 0.8316 & 0.8978 & 0.0209 \\
\hline & $P_{\text {interaction }}$ & & & & & & 0.0607 \\
\hline \multirow[t]{4}{*}{ LEA (ng/ml) } & $\mathrm{CC}$ & $0 \cdot 7448$ & $0 \cdot 7416$ & 0.6877 & 0.8598 & 0.9128 & 0.7893 \\
\hline & CA/AA & 0.9668 & 0.8751 & 0.7295 & 0.9981 & 1.0449 & 0.9229 \\
\hline & $P$ & 0.0346 & 0.6084 & 0.9998 & 0.5640 & 0.6273 & 0.0094 \\
\hline & $P_{\text {interaction }}$ & & & & & & 0.1392 \\
\hline \multirow[t]{4}{*}{ AEA (ng/ml) } & CC & 0.4447 & 0.4947 & 0.4295 & 0.4325 & 0.4358 & 0.4474 \\
\hline & CA/AA & 0.5156 & 0.5273 & 0.4579 & 0.4817 & 0.4966 & 0.4958 \\
\hline & $P$ & 0.5242 & 0.9937 & 0.9978 & 0.9088 & 0.7308 & 0.0716 \\
\hline & $P_{\text {interaction }}$ & & & & & & 0.6260 \\
\hline \multirow[t]{4}{*}{ ALEA (ng/ml) } & $\mathrm{CC}$ & 0.0457 & 0.0401 & 0.0403 & 0.0946 & 0.0387 & 0.0519 \\
\hline & CA/AA & 0.0647 & 0.0486 & 0.0426 & 0.1162 & 0.0441 & 0.0632 \\
\hline & $P$ & 0.0832 & 0.9430 & 1.0000 & 0.0257 & 0.9978 & 0.0073 \\
\hline & $P_{\text {interaction }}$ & & & & & & 0.0337 \\
\hline \multirow[t]{4}{*}{ DHEA (ng/ml) } & $\mathrm{CC}$ & 0.9742 & 1.0427 & 1.6906 & 0.9871 & 0.9401 & $1 \cdot 1270$ \\
\hline & CA/AA & 1.2361 & $1 \cdot 1554$ & $2 \cdot 2018$ & 1.0901 & 1.0608 & 1.3488 \\
\hline & $P$ & 0.0848 & 0.9561 & $<0.0001$ & 0.9761 & 0.9336 & 0.0002 \\
\hline & $P_{\text {interaction }}$ & & & & & & 0.0001 \\
\hline
\end{tabular}

Canola, conventional canola oil; CanolaOleic, high oleic canola oil; CanolaDHA, DHA-enriched canola oil; FlaxSaff, a blend of flax oil and safflower oil; CornSaff, a blend of corn oil and safflower oil; PEA, palmitoylethanolamide; OEA, oleoylethanolamide; LEA, linoleoylethanolamide; AEA, arachidonoylethanolamine; ALEA, $a$-linolenoylethanolamide; DHEA, docosahexaenoylethanolamide.

* Significant difference: $P<0.05$. $P$ value shows the differences between two genotypes within the same treatment. $P_{\text {interaction }}$ shows the gene-treatment interaction in the entire population.

circulating pool, the enzyme activities of FAE synthesis and degradation are more crucial for circulating FAE levels ${ }^{(15,23)}$. As such, our important findings demonstrate that the fat types in the diet can positively influence the corresponding FAE levels in humans. These results also provide evidence supporting the conclusion that dietary FA lead to increased circulating FAE in both piglets ${ }^{(12)}$ and humans ${ }^{(36,48)}$, although it remains to be established whether such changes in plasma FAE levels further alter the FAE levels in target tissues such as brain, liver and intestines.

Polyunsaturated long-chain FAE are a relatively minor group compared with saturated and monounsaturated FAE, such as PEA and OEA, which are the major FAE in plasma 
and tissues ${ }^{(49)}$. Evidence shows that feeding a high-SFA diet to mice $^{(50)}$ and rats ${ }^{(51)}$ resulted in lower levels of PEA and OEA. Joosten et al. ${ }^{(48)}$ reported that the two most abundant FAE, PEA and OEA levels, were approximately 7-fold higher than AEA levels in a clinical study. Our observation concurs with the findings with similar absolute values for PEA and OEA ranging from $0 \cdot 1$ to $4.0 \mathrm{ng} / \mathrm{ml}$. Owing to the intervention design in our clinical trial, we were unable to demonstrate the effect of dietary SFA on PEA and OEA. However, we noticed that although post-treatment plasma LA, LEA precursor, levels were higher than OA, OEA precursor, levels in our population, the overall plasma LEA levels were lower than OEA. The correlation coefficient between LA and LEA was less significant than that between OA and OEA. It is possible that the endogenous conversion from OA to OEA may be faster compared with LA to LEA. One possible explanation is because of the variations in the positional distribution of FA on TAG molecules. The committed step of FAE synthesis is the exchange of FA at the stereospecific numbering-1 (sn-1) position. Usually, the sn-1 position contains a SFA, whereas the sn-2 position more likely is occupied by an unsaturated FA. Accordingly, PEA levels are overall the highest among the six FAE of interest in our observation as well as in other similar studies. In mammals, the ratio of OA:LA at the sn-1 position is higher than the ratio of LA: OA, which can potentially lead to the higher synthesis rate of OEA compared with LEA. In addition, the negative correlations between android fat mass changes and FAE (OEA, LEA and AEA) indicated that these endogenous FAE levels may be involved in the regulation of food intake and energy balance as suggested by Diep et al. ${ }^{(51)}$.

This study has strengths and limitations. First, in contrast to a few previous human studies that relied on small subject numbers on the post-treatment FAE response, this study was powered to examine a larger group of people in North America. Furthermore, to the best of our knowledge, this is the first study ever to show that DHEA may have a suppressive effect on OEA levels, potentially resulting in a failure in body fat loss. Unfortunately, we were unable to assess the baseline FAE contents in plasma, which can establish the initial FAE profiles of the participants for further comparison. Although the cross-over design of our trial clearly showed the treatment effects on different circulating FAE levels, lacking of baseline values restricted our prediction on the association between change of FAE and change of body fat mass in response to different oil treatments. Nonetheless, the correlation analysis was only performed in a small subgroup of twenty-seven subjects with an unbalanced sex ratio due to the limited design of satellite sites in such multicentre trial, and thus our attempt was not able to assess the sex effects, and the question of how the shifts of FAE influence the body fat response requires further investigation.

In summary, our study reports several novel findings that clarify our understanding of the metabolic and physiological responses associated with different dietary FA classes. Our finding on the genetic variants indicates that the polymorphism rs324420 in FAAH may influence the beneficial function of FAE in humans by altering the circulating FAE levels. Furthermore, the elevated circulating DHEA in response to dietary DHA may suppress OEA levels and, in turn, interfere with the function of OEA, which may disrupt regulation of body weight.

\section{Acknowledgements}

The authors are indebted to Haifeng Yang for optimising the performance of the UPLS and GC-FID. The authors also acknowledge Dennis Labossiere for his interpretation of the plasma FA chromatograph. The authors especially thank the personnel at all participating centres, including investigators, clinical coordinators, kitchen staff, analytical teams and study volunteers.

This study was conducted under the clinical project of COMIT, which was supported by the Canola Council of Canada, Flax Council of Canada, Agriculture and Agri-Food Canada and Dow AgroSciences, Canada Research Chairs and Western Grains Research Foundation through the Growing Forward programme of Agriculture and Agri-Food Canada. The present study was also supported by the Manitoba's Agri-Food Research and Development Initiative (ARDI) (ID: GRW FWD ARDI\#12-1186).

The authors' contributions are as follows: S. P. and P. J. H. J. contributed to the design of the study and wrote the manuscript. S. P. and X. L. conducted the data analyses. S. P., P. E. and P. J. H. J. were responsible for interpretation of the results. D. J. A. J., P. W. C., B. L., P. M. E.-K., S. G. W. and P. J. H. J. provided supervision.

None of the authors has any conflicts of interest to declare.

\section{Supplementary material}

For supplementary material/s referred to in this article, please visit http://dx.doi.org/doi:10.1017/S0007114515005425

\section{References}

1. Epps DE, Natarajan V, Schmid PC, et al. (1980) Accumulation of $\mathrm{N}$-acylethanolamine glycerophospholipids in infarcted myocardium. Biochim Biophys Acta 618, 420-430.

2. Devane WA, Hanus L, Breuer A, et al. (1992) Isolation and structure of a brain constituent that binds to the cannabinoid receptor. Science 258, 1946-1949.

3. Marczylo TH, Lam PM, Amoako AA, et al. (2010) Anandamide levels in human female reproductive tissues: solid-phase extraction and measurement by ultraperformance liquid chromatography tandem mass spectrometry. Anal Biochem 400, 155-162.

4. Pasquariello N, Catanzaro G, Marzano V, et al. (2009) Characterization of the endocannabinoid system in human neuronal cells and proteomic analysis of anandamide-induced apoptosis. J Biol Chem 284, 29413-29426.

5. Pacher P, Batkai S \& Kunos G (2006) The endocannabinoid system as an emerging target of pharmacotherapy. Pharmacol Rev 58, 389-462.

6. Romano A, Coccurello R, Giacovazzo G, et al. (2014) Oleoylethanolamide: a novel potential pharmacological alternative to cannabinoid antagonists for the control of appetite. Biomed Res Int 2014, 203425.

7. Fu J, Gaetani S, Oveisi F, et al. (2003) Oleylethanolamide regulates feeding and body weight through activation of the nuclear receptor PPAR-alpha. Nature 425, 90-93.

8. Lo Verme J, Gaetani S, Fu J, et al. (2005) Regulation of food intake by oleoylethanolamide. Cell Mol Life Sci 62, 708-716. 
9. Hoareau L, Buyse M, Festy F, et al. (2009) Anti-inflammatory effect of palmitoylethanolamide on human adipocytes. Obesity (Silver Spring) 17, 431-438.

10. Klein TW (2005) Cannabinoid-based drugs as antiinflammatory therapeutics. Nat Rev Immunol 5, 400-411.

11. O'Sullivan SE (2007) Cannabinoids go nuclear: evidence for activation of peroxisome proliferator-activated receptors. $\mathrm{Br} \mathrm{J}$ Pharmacol 152, 576-582.

12. Berger A, Crozier G, Bisogno T, et al. (2001) Anandamide and diet: inclusion of dietary arachidonate and docosahexaenoate leads to increased brain levels of the corresponding $\mathrm{N}$-acylethanolamines in piglets. Proc Natl Acad Sci U S A 98 , 6402-6406.

13. Kim HY, Moon HS, Cao D, et al. (2011) $\mathrm{N}$ docosahexaenoylethanolamide promotes development of hippocampal neurons. Biochem J 435, 327-336.

14. Rahman IA, Tsuboi K, Uyama T, et al. (2014) New players in the fatty acyl ethanolamide metabolism. Pharmacol Res 86, 1-10

15. Tsuboi K, Ikematsu N, Uyama T, et al. (2013) Biosynthetic pathways of bioactive $N$-acylethanolamines in brain. CNS Neurol Disord Drug Targets 12, 7-16.

16. Thabuis C, Tissot-Favre D, Bezelgues JB, et al. (2008) Biological functions and metabolism of oleoylethanolamide. Lipids 43, 887-894.

17. Sipe JC, Waalen J, Gerber A, et al. (2005) Overweight and obesity associated with a missense polymorphism in fatty acid amide hydrolase (FAAH). Int J Obes (Lond) 29, 755-759.

18. Monteleone P, Tortorella A, Martiadis V, et al. (2008) The cDNA 385C to A missense polymorphism of the endocannabinoid degrading enzyme fatty acid amide hydrolase (FAAH) is associated with overweight/obesity but not with binge eating disorder in overweight/obese women. Psychoneuroendocrinology 33, 546-550.

19. Jensen DP, Andreasen CH, Andersen MK, et al. (2007) The functional Pro129Thr variant of the $F A A H$ gene is not associated with various fat accumulation phenotypes in a population-based cohort of 5801 whites. J Mol Med 85, 445-449.

20. Monteleone P, Milano W, Petrella C, et al. (2010) Endocannabinoid Pro129Thr FAAH functional polymorphism but not 1359G/A CNR1 polymorphism is associated with antipsychotic-induced weight gain. J Clin Psychopharmacol 30, 441-445.

21. Hariri AR, Gorka A, Hyde LW, et al. (2009) Divergent effects of genetic variation in endocannabinoid signaling on human threatand reward-related brain function. Biol Psychiatry 66, 9-16.

22. Wangensteen T, Akselsen H, Holmen J, et al. (2011) A common haplotype in NAPEPLD is associated with severe obesity in a Norwegian population-based cohort (the HUNT study). Obesity (Silver Spring) 19, 612-617.

23. Geurts L, Everard A, Van Hul M, et al. (2015) Adipose tissue NAPE-PLD controls fat mass development by altering the browning process and gut microbiota. Nat Commun 6, 6495.

24. Piers LS, Walker KZ, Stoney RM, et al. (2003) Substitution of saturated with monounsaturated fat in a 4 -week diet affects body weight and composition of overweight and obese men. Br J Nutr 90, 717-727.

25. Moussavi N, Gavino V \& Receveur O (2008) Could the quality of dietary fat, and not just its quantity, be related to risk of obesity? Obesity (Silver Spring) 16, 7-15.

26. Pelkman CL, Fishell VK, Maddox DH, et al. (2004) Effects of moderate-fat (from monounsaturated fat) and low-fat weightloss diets on the serum lipid profile in overweight and obese men and women. Am J Clin Nutr 79, 204-212.

27. Krishnan S \& Cooper JA (2014) Effect of dietary fatty acid composition on substrate utilization and body weight maintenance in humans. Eur J Nutr 53, 691-710.
28. Naughton SS, Mathai ML, Hryciw DH, et al. (2013) Fatty Acid modulation of the endocannabinoid system and the effect on food intake and metabolism. Int J Endocrinol 2013, 361895.

29. Jones PJ, Senanayake VK, Pu S, et al. (2014) DHA-enriched high-oleic acid canola oil improves lipid profile and lowers predicted cardiovascular disease risk in the canola oil multicenter randomized controlled trial. Am J Clin Nutr 100, 88-97.

30. Senanayake VK, Pu S, Jenkins DA, et al. (2014) Plasma fatty acid changes following consumption of dietary oils containing $n-3, n-6$, and $n-9$ fatty acids at different proportions: preliminary findings of the Canola oil Multicenter Intervention Trial (COMIT). Trials 15, 136.

31. Mifflin MD, St Jeor ST, Hill LA, et al. (1990) A new predictive equation for resting energy expenditure in healthy individuals. Am J Clin Nutr 51, 241-247.

32. Folch J, Lees M \& Sloane Stanley GH (1957) A simple method for the isolation and purification of total lipides from animal tissues. J Biol Chem 226, 497-509.

33. Lin L, Yang H \& Jones PJ (2012) Quantitative analysis of multiple fatty acid ethanolamides using ultra-performance liquid chromatography-tandem mass spectrometry. Prostaglandins Leukot Essent Fatty Acids 87, 189-195.

34. Marczylo TH, Lam PM, Nallendran V, et al. (2009) A solidphase method for the extraction and measurement of anandamide from multiple human biomatrices. Anal Biochem 384, 106-113.

35. Lin L, Rideout T, Yurkova N, et al. (2013) Fatty acid ethanolamides modulate CD36-mRNA through dietary fatty acid manipulation in Syrian Golden hamsters. Appl Physiol Nutr Metab 38, 870-878.

36. Jones PJ, Lin L, Gillingham LG, et al. (2014) Modulation of plasma $\mathrm{N}$-acylethanolamine levels and physiological parameters by dietary fatty acid composition in humans. J Lipid Res $\mathbf{5 5}$, 2655-2664.

37. Pecina M, Martinez-Jauand M, Hodgkinson C, et al. (2014) FAAH selectively influences placebo effects. Mol Psychiatry 19, 385-391.

38. Tsuboi K, Okamoto Y, Ikematsu N, et al. (2011) Enzymatic formation of $\mathrm{N}$-acylethanolamines from $\mathrm{N}$-acylethanolamine plasmalogen through $N$-acylphosphatidylethanolaminehydrolyzing phospholipase D-dependent and -independent pathways. Biochim Biophys Acta 1811, 565-577.

39. Simon GM \& Cravatt BF (2010) Characterization of mice lacking candidate $\mathrm{N}$-acyl ethanolamine biosynthetic enzymes provides evidence for multiple pathways that contribute to endocannabinoid production in vivo. Mol Biosyst 6, 1411-1418.

40. Blankman JL \& Cravatt BF (2013) Chemical probes of endocannabinoid metabolism. Pharmacol Rev $\mathbf{6 5}$, 849-871.

41. Muller TD, Bronner G, Wandolski M, et al. (2010) Mutation screen and association studies for the fatty acid amide hydrolase $(F A A H)$ gene and early onset and adult obesity. BMC Med Gene 11, 2.

42. Yang R, Fredman G, Krishnamoorthy S, et al. (2011) Decoding functional metabolomics with docosahexaenoyl ethanolamide (DHEA) identifies novel bioactive signals. J Biol Chem 286, 31532-31541.

43. Brown I, Cascio MG, Wahle KW, et al. (2010) Cannabinoid receptor-dependent and -independent anti-proliferative effects of omega-3 ethanolamides in androgen receptorpositive and -negative prostate cancer cell lines. Carcinogenesis 31, 1584-1591.

44. Wood JT, Williams JS, Pandarinathan L, et al. (2010) Dietary docosahexaenoic acid supplementation alters select physiological endocannabinoid-system metabolites in brain and plasma. J Lipid Res 51, 1416-1423. 
45. Rashid MA, Katakura M, Kharebava G, et al. (2013) Ndocosahexaenoylethanolamine is a potent neurogenic factor for neural stem cell differentiation. J Neurochem 125, 869-884.

46. Ramsden CE, Zamora D, Makriyannis A, et al. (2015) Diet-induced changes in $n$-3- and $n$-6-derived endocannabinoids and reductions in headache pain and psychological distress.J Pain 16, 707-716.

47. Rodriguez de Fonseca F, Navarro M, Gomez R, et al. (2001) An anorexic lipid mediator regulated by feeding. Nature $\mathbf{4 1 4}$, 209-212.

48. Joosten MM, Balvers MG, Verhoeckx KC, et al. (2010) Plasma anandamide and other $\mathrm{N}$-acylethanolamines are correlated with their corresponding free fatty acid levels under both fasting and non-fasting conditions in women. Nutr Metab (Lond) 7, 49.

49. Schmid HH, Schmid PC \& Berdyshev EV (2002) Cell signaling by endocannabinoids and their congeners: questions of selectivity and other challenges. Chem Phys Lipids 121, 111-134.

50. Aviello G, Matias I, Capasso R, et al. (2008) Inhibitory effect of the anorexic compound oleoylethanolamide on gastric emptying in control and overweight mice. J Mol Med (Berl) 86, 413-422.

51. Diep TA, Madsen AN, Holst B, et al. (2011) Dietary fat decreases intestinal levels of the anorectic lipids through a fat sensor. FASEB J 25, 765-774. 